\title{
AN EQUILIBRIUM APPROACH FOR FLEXURAL-TORSIONAL BUCKLING OF ELASTIC STEEL ARCHES
}

\author{
Y.L. $\mathrm{Pi}^{1}$, M.A. Bradford and Y.Y. Chen \\ ${ }^{1}$ The corresponding author, Senior Research Fellow, School of Civil and Environmental Engineering, \\ NSW, Sydney, NSW 2052, Australia. \\ Email: y.pi@unsw.edu.au
}

\begin{abstract}
When an arch is subjected to in-plane loading, it may suddenly deflect laterally and twist out of the plane of loading and fail in a flexural-torsional buckling mode. This paper presents a static equilibrium approach for the elastic flexural-torsional buckling of circular arches under uniform bending, or under uniform compression. Solutions for the buckling moment and buckling load are obtained in closed form, and discrepancies among existing solutions are clarified. It is found that it is reasonable to use material curvatures and twist, rather than spatial curvatures and twist, for the flexural-torsional buckling analysis of arches. First order buckling deformations provide a sufficient basis for the static equilibrium methods for the flexural-torsional buckling analysis of arches. Equilibrium of a lengthwise differential element of an arch should be considered in the analysis, and so the couplings between the lateral bending and torsional actions and resistances can be included in the differential equations of equilibrium. If the equilibrium is considered only at the cross-section, this is equivalent to treating arches as analogous with straight members, and so the coupling terms in the differential equations of equilibrium are lost.
\end{abstract}

Keywords: Arch, Bending, Buckling, Compression, Elastic, Equilibrium Approach, Flexural-Torsional

\section{INTRODUCTION}

When an arch is subjected to in-plane loading, it may suddenly deflect laterally and twist out of the plane of loading and fail in a flexural-torsional buckling mode. In general, finite element methods can be used to obtain the flexural-torsional buckling load under general conditions of in-plane loading and restraints. When a circular arch is subjected to in-plane uniform bending or to uniform compression (Figures 1(a) and 1(b)), closed form solutions for the elastic flexural-torsional buckling (Figs 1(c)(d)) can be obtained, and the buckling loads for an arch under uniform bending or uniform compression are important reference loads for the design of a steel arch [1]. The elastic flexural-torsional buckling of arches under uniform bending or under uniform compression has been studied extensively by a number of researchers [2-9] using energy or virtual work approaches. Energy and virtual approaches are suitable for systematic treatments. However, because energy and virtual approaches usually deal with strains and stress resultants, second order nonlinear strain-displacement relationships are needed [10]. Different methods are used in deriving the strain-displacement relationships [2-9] and so different second order terms for strain-displacement relationships can be obtained. As a result, the closed form solutions for flexural-torsional buckling loads derived by different researchers may differ from each other.

Static equilibrium approaches for flexural-torsional buckling analysis deal with equilibrium between external loads and internal resistances directly, and thus the second order strain-displacement relationships are not needed. In addition, since the buckled equilibrium configuration is infinitesimally close to the unbuckled equilibrium configuration, the curvatures and the buckling actions related to the first order buckling deformations are sufficient for the buckling analysis. Static equilibrium approaches have been used only by Timoshenko and Gere [2] for arches under uniform bending, and by Vlasov [11] for arches under uniform bending or under uniform compression. Timoshenko and Gere [2] considered equilibrium at the cross-section to establish the differential equations of equilibrium in the same way as for straight beams. However, in an arch, the lateral bending action and resistance are coupled with the torsional action and resistance, so that 
neither the lateral bending action and resistance nor the torsion action and resistance alone are in equilibrium. Equilibrium of a lengthwise differential element of an arch, rather than only at the cross-section, should therefore be considered, and so the method of Timoshenko and Gere [2] appears to miss the coupling terms in the differential equations of equilibrium for the buckling deformations. Vlasov [11] substituted the spatial curvatures and twist into the differential equations of equilibrium that were derived for the flexural-torsional buckling analysis of straight members. In general, however, this method is not suitable for the flexural-torsional buckling of arches, and it has been shown [4-9] that the method may lead to an incorrect flexural-torsional buckling load for arches under uniform compression.

The purpose of this paper is to use a static equilibrium approach to study the elastic flexural-torsional buckling of circular arches with doubly symmetric cross-section under uniform bending or under uniform compression, to obtain closed form solutions for the buckling moment and load, and to clarify the discrepancies that arise among existing solutions.

\section{SPATIAL AND MATERIAL CURVATURES}

The following assumptions are made in this study: (1) the Euler-Bernolli bending theory and Vlasov's torsion theory are used, so that the cross-section is assumed to remain rigid and local buckling and/or effects of distortion of the cross-section are not considered; (2) the arches are circular and of doubly symmetric uniform cross-section, so that the centroid and the shear centre of the cross-section coincide; (3) the height $D$ of the cross-section is much smaller than both the length $S$ and the radius $R$ of initial curvature of the arch, i.e. $D / S<<1$ and $D / R<<1$, which imply that the arches considered are slender; and (4) the strains are small.

A body attached curvilinear orthogonal axis system oxys is defined as follows. The axis os passes through the locus of the centroids of the cross-section of the arch and the axes $o x$ and $o y$ coincide with the principal axes of the cross-section, as shown in Figures 1-3. In the undeformed configuration, the axis $o y$ is towards to the centre of the arch. After deformation, the origin $o$ displaces $u, v, w$ in the directions of the axes $o x, o y$, and $o s$ to $o_{1}$ and the cross-sections (that are assumed to remain rigid in their plane and so do not distort) rotate through an angle $\phi$, and so the body attached curvilinear orthogonal axis system oxys moves and rotates to a new position $o_{1} x_{1} y_{1} s_{1}$ as shown in Figure 2. In general, the centroidal axis os of a circular arch has an initial curvature $\kappa_{x 0}$ about the major principal axis $o x$ (i.e. in the direction of the axis $o y$ ) as shown in Figure 1).

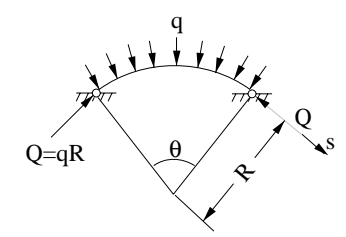

(a) Arch in uniform compression

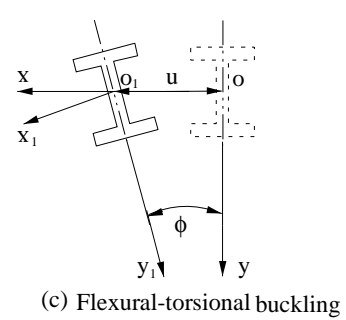

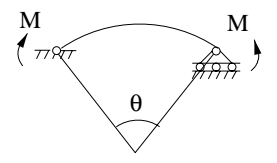

(b) Arch in uniform bending

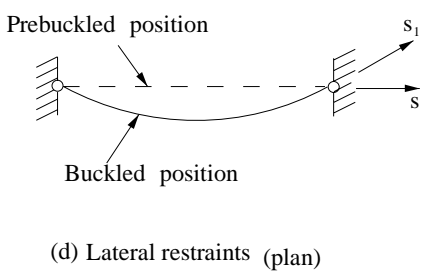

Figure 1. Flexural-torsional buckling of arches 
A basis vector $\mathbf{p}_{s}$ in the tangent direction of the axis $o$, and basis vectors $\mathbf{p}_{x}$ and $\mathbf{p}_{y}$ in the directions of the axes ox and oy (Figures 2-3) are used as the fixed reference basis. The basis vectors $\mathbf{p}_{x}, \mathbf{p}_{y}$, and $\mathbf{p}_{s}$ do not change with the deformation, but their directions change from point to point along the arch axis os. In the deformed configuration, a basis vector $\mathbf{q}_{s}$ is defined in the tangent direction of the axis $o_{1} s_{1}$, and basis vectors $\mathbf{q}_{x}$ and $\mathbf{q}_{y}$ are defined in the direction of the principal axes $o_{1} x_{1}, o_{1} y_{1}$ of the rotated cross-section at $o_{1}$ as shown in Figures 2-3. The basis vectors $\mathbf{q}_{x}, \mathbf{q}_{y}, \mathbf{q}_{s}$ are attached to the arch and move with the arch during the deformation with the vector $\mathbf{q}_{s}$ being normal to the cross-section at all times.

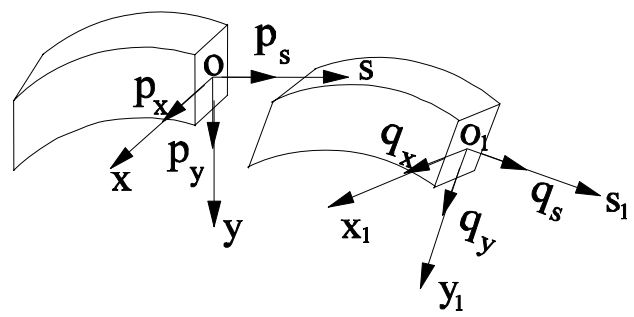

Figure 2. Basis vectors before and after buckling
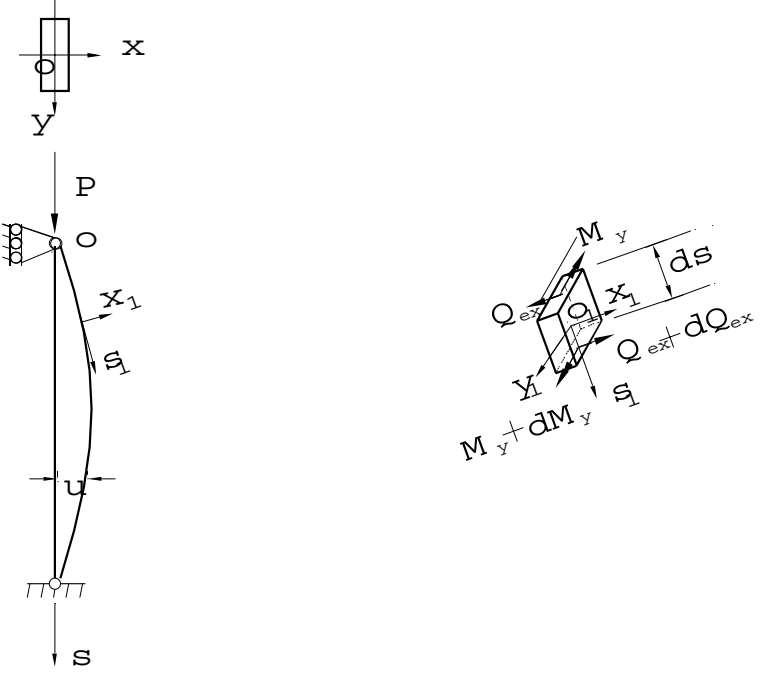

(a) Laterial buckling of column

(b) Alternative method

Figure 3. Lateral buckling of a column under uniform compression

The rotation from the basis vectors $\mathbf{p}_{x}, \mathbf{p}_{y}, \mathbf{p}_{s}$ to the basis vectors $\mathbf{q}_{x}, \mathbf{q}_{y}, \mathbf{q}_{s}$ (Figure 2) can be expressed using a rotation matrix $\mathbf{R}$ as [12-13]

$$
\mathbf{q}_{i}=\mathbf{R p}_{i} \quad(i=x, y, s)
$$

Static equilibrium methods deal with the equilibrium of external loads and internal resistances directly, and it is well known that the classical flexural-torsional buckling is characterized by the fact that, as the load passes through its critical stage, the structure passes from its unbuckled equilibrium configuration to an infinitesimally close buckled equilibrium configuration, Therefore, 
second order terms are not needed in the flexural-torsional buckling analysis for arches by static equilibrium approaches, and so a small-rotation matrix $\mathbf{R}$ is sufficient for undertaking the buckling analysis, which is given by

$$
\mathbf{R}=\left[\begin{array}{ccc}
1 & -\phi & u^{\prime} \\
\phi & 1 & \tilde{v}^{\prime} \\
-u^{\prime} & -\tilde{v}^{\prime} & 1
\end{array}\right]
$$

where $\tilde{v}^{\prime}=v^{\prime}-w \kappa_{x 0},()^{\prime}=\mathrm{d}() / \mathrm{d} s$, and the initial curvature $\kappa_{x 0}$ of the centroidal axis os of a circular is given by $\kappa_{x 0}=-1 / R$ for the arches with the upward rise as shown in Figure 1.

It can be obtained from calculus of vectors [14] that the spatial curvatures referred to the undeformed configuration and the material curvatures referred to the deformed configuration can be obtained from the rotation matrix $\mathbf{R}$ as

$$
\boldsymbol{\Omega}=\frac{\mathrm{d} \mathbf{R}}{\mathrm{d} s} \mathbf{R}^{T}+\mathbf{R} \mathbf{K}_{0} \mathbf{R}^{T} \text { and } \mathbf{K}=\mathbf{R}^{T} \frac{\mathrm{d} \mathbf{R}}{\mathrm{d} s}+\mathbf{R}^{T} \mathbf{K}_{0} \mathbf{R}
$$

where ( $)^{T}$ is the transpose of a matrix, $\mathbf{K}_{0}$ is the matrix of the initial curvatures and given by

$$
\mathbf{K}_{0}=\left[\begin{array}{ccc}
0 & 0 & 0 \\
0 & 0 & -\kappa_{x 0} \\
0 & \kappa_{x 0} & 0
\end{array}\right]
$$

while $\boldsymbol{\Omega}$ and $\mathbf{K}$ are the matrices of spatial and material curvatures and given by

$$
\boldsymbol{\Omega}=\left[\begin{array}{ccc}
0 & -\omega_{s} & \omega_{y} \\
\omega_{s} & 0 & -\omega_{x} \\
-\omega_{y} & \omega_{x} & 0
\end{array}\right] \text { and } \mathbf{K}=\left[\begin{array}{ccc}
0 & -\kappa_{s} & \kappa_{y} \\
\kappa_{s} & 0 & -\kappa_{x} \\
-\kappa_{y} & \kappa_{x} & 0
\end{array}\right]
$$

in which $\omega_{x}$ and $\omega_{y}$ are the spatial curvatures about the positive direction of the axes $o x$ and $o y$, and $\omega_{s}$ is the spatial twist about the positive direction of the os axis in the undeformed configuration; while $\kappa_{x}$ and $\kappa_{y}$ are the material curvatures about the positive direction of the axes $o_{1} x_{1}$ and $o_{1} y_{1}$, and $\kappa_{s}$ is the material twist about the positive direction of the $o_{1} s_{1}$ axis in the deformed configuration.

Substituting Eqs. (2) and (4) into Eq. (3) and ignoring second and higher order terms leads to the spatial curvatures $\omega_{x}$ and $\omega_{y}$, and the spatial twist $\omega_{s}$ as

$$
\begin{aligned}
& \omega_{x} \approx-\tilde{v}^{\prime \prime}+\kappa_{x 0}=-\tilde{v}^{\prime \prime}-\frac{1}{R} \\
& \omega_{y} \approx u^{\prime \prime}+\phi \kappa_{x 0}=u^{\prime \prime}-\frac{\phi}{R} \\
& \omega_{s} \approx \phi^{\prime}-u^{\prime} \kappa_{x 0}=\phi^{\prime}+\frac{u^{\prime}}{R}
\end{aligned}
$$

and to the material curvatures $\kappa_{x}$ and $\kappa_{y}$ and the material twist $\kappa_{s}$ as

$$
\kappa_{x} \approx-\tilde{v}^{\prime \prime}+\kappa_{x 0}=-\tilde{v}^{\prime \prime}-\frac{1}{R}
$$




$$
\begin{aligned}
& \kappa_{y} \approx u^{\prime \prime}-\phi \kappa_{x 0}=u^{\prime \prime}+\frac{\phi}{R} \\
& \kappa_{s} \approx \phi^{\prime}+u^{\prime} \kappa_{x 0}=\phi^{\prime}-\frac{u^{\prime}}{R}
\end{aligned}
$$

where ()$^{\prime \prime} \equiv \mathrm{d}^{2}() / \mathrm{d} s^{2}$.

In studies of the flexural-torsional buckling of an arch, the spatial curvatures and twist given by Eqs. (7)-(9) have been used by a number of researchers [2-3,5,11] while the material curvatures given by Eqs. (10)-(12) have been used by other researchers [4,6-9]. It is noteworthy that the equilibrium for flexural-torsional buckling of an arch should be referred to the buckled configuration. Hence, it is reasonable to use the material curvatures and twist rather than the spatial curvatures and twist for the flexural-torsional buckling analysis of arches.

\section{UNIFORM COMPRESSION}

\subsection{Buckling of Columns}

In order to compare the differences between the flexural-torsional buckling of arches and the buckling of columns under uniform compression, the buckling of a column under uniform compression is briefly summarized in the following. In the buckled configuration, the axial compressive force $P$ has the components: a lateral force $Q_{e x}$ in the direction of the axis $o_{1} x_{1}$, a lateral force $Q_{e y}$ in the direction of the axis $o_{1} y_{1}$, and an axial force $Q_{e s}$ in the direction of the axis $o_{1} \mathrm{~s}_{1}$ and they are given by

$$
\left\{\begin{array}{l}
Q_{e x} \\
Q_{e y} \\
Q_{e s}
\end{array}\right\}=\mathbf{R}^{T}\left\{\begin{array}{l}
0 \\
0 \\
P
\end{array}\right\}=\left\{\begin{array}{c}
-P u^{\prime} \\
-P v^{\prime} \\
P
\end{array}\right\}
$$

where the rotation matrix $\mathbf{R}$ is the same as that given by Eq. (2) with $\tilde{v}^{\prime}=v^{\prime}$.

The elastic lateral buckling load of a column about its minor principal axis oy can be determined by considering lateral equilibrium in the buckled configuration as shown in Figure 3. Two methods can be used to obtain the buckling load. Most researchers $[2,10,15]$ have used the method shown in Figure 3(a) and have obtained the differential equation of equilibrium for the lateral buckling as

$$
P u+E I_{y} u^{\prime \prime}=0
$$

where $E$ is Young's modulus of elasticity and $I_{y}$ is the second moment of area of the cross-section about its minor principal axis $o y$.

Alternatively, the differential equation of equilibrium for lateral buckling can be obtained by considering the lateral equilibrium of a differential element of length $\mathrm{d} s$ in the buckled configuration shown in Figure 3(b). For lateral bending equilibrium,

$$
\mathrm{d} M_{y}+Q_{e x} \mathrm{~d} s=0
$$


where the bending moment $M_{y}$ about the axis $o_{1} y_{1}$ is equal to the internal bending resistance of the cross-section $M_{i y}$ and so given by [15] $M_{y}=M_{i y}=E I_{y} u^{\prime \prime}$; and $Q_{e x}$ is the lateral component of the axial load $P$ in the buckled configuration given by Eq. (13). Hence, Eq. (15) becomes

$$
P u^{\prime}+E I_{y} u^{\prime \prime \prime}=0
$$

The solution that satisfies the kinematic boundary conditions $u_{0}=u_{S}=0$ can be assumed to be given by

$$
\frac{u}{u_{m}}=\sin \frac{n \pi s}{S}
$$

where $u_{m}$ is the maximum lateral displacement during buckling, $s$ is the coordinate in the column axis os as shown in Figure 3, $S$ is the length of the column and $n$ is the number of the buckled half waves. Substituting Eq. (17) into Eq. (14) or (16) leads to the $n$-th mode elastic flexural buckling load of a column about its minor principal axis of the cross-section $[2,15]$ given by

$$
P_{y n}=\frac{(n \pi)^{2} E I_{y}}{S^{2}}
$$

The axial compressive force $Q_{e s}$ in the direction of the axis $o_{1} s_{1}$ may produce an external torque about the axis $o_{1} s_{1}$ and so the column may buckle in a torsional mode. Consider the differential element of length $\mathrm{d} s$ and differential strip $\mathrm{d} A \mathrm{~d} s$ as shown in Figure 4, where $\mathrm{d} A$ is the cross-sectional area of the differential strip. The relative twist angle between both ends can be represented by $\mathrm{d} \phi$ (Figure 4(b)). The force acting on the differential area $\mathrm{d} A$ due to the axial compressive force $Q_{e s}$ is equal to $\mathrm{d} q_{e s}=\left(Q_{e s} / A\right) \mathrm{d} A$, where $A$ is the area of the cross-section. The distance of the differential area $\mathrm{d} A$ from the shear centre of the cross-section is given by $\rho=\sqrt{x^{2}+y^{2}}$ (Figure 4(a)) and the relative displacement of both ends of the differential strip due to the relative twist angle $\mathrm{d} \phi$ is equal to $\rho \mathrm{d} \phi$. Hence, the force $\mathrm{d} q_{e s}$ has a component $\mathrm{d} q=\mathrm{d} q_{e s}(\rho \mathrm{d} \phi / \mathrm{d} s)$ (Figure 4(b)) which exerts a torque $\mathrm{d} m_{e s}$ about the shear centre given by $\mathrm{d} m_{e s}=\mathrm{d} q \times \rho=\mathrm{d} q_{e s} \rho^{2}(\mathrm{~d} \phi / \mathrm{d} s)$, and so the total external torque $M_{e s}$ produced by the axial compressive force $Q_{e s}$ is

$$
M_{e s}=\int_{A} d m_{e s}=\int_{A} d q_{e s} \rho^{2} \frac{d \phi}{d s}=\int_{A} \frac{Q_{e s}}{A} \rho^{2} \frac{d \phi}{d s} d A=r_{0}^{2} P \phi^{\prime}
$$

where $r_{0}$ is the radius of gyration of the cross-section and given by

$$
r_{0}=\sqrt{\frac{\int_{A} \rho^{2} \mathrm{~d} A}{\mathrm{~A}}}=\sqrt{\frac{\int_{A}\left(x^{2}+\mathrm{y}^{2}\right) \mathrm{d} A}{\mathrm{~A}}}=\sqrt{\frac{I_{x}+I_{y}}{\mathrm{~A}}}
$$

and in which $I_{x}$ is the second moment of area of the cross-section about its major principal axis $o x$.

The internal torsional resistance is given by [15]

$$
M_{i s}=-G J \phi^{\prime}+E I_{w} \phi^{\prime \prime \prime}
$$


where $G$ is the elastic shear modulus, and $J$ and $I_{w}$ re the torsion and warping constants of the cross-section. The axial force $Q_{e s}=P$ is given by Eq. (13). During torsional buckling, the resultant of the external and internal torques vanishes and so

$$
M_{e s}+M_{i s}=r_{0}^{2} P \phi^{\prime}-G J \phi^{\prime}+E I_{w} \phi^{\prime \prime \prime}=0
$$

which is the differential equation of equilibrium for the torsional buckling of a column under uniform compression [2,15].

The solution that satisfies the kinematic boundary conditions $\phi_{0}=\phi_{S}=0$ can be assumed to be given by

$$
\frac{\phi}{\phi_{m}}=\sin \frac{n \pi s}{S}
$$

where $\phi_{m}$ is the maximum twist angle of the cross-section during buckling. Substituting Eq. (23) into Eq. (22) leads to the $n$-th mode elastic torsional buckling load of a column about its shear centre axis [2,15]given by

$$
P_{s n}=\frac{1}{r_{0}^{2}}\left(G J+\frac{(n \pi)^{2} E I_{w}}{S^{2}}\right)
$$

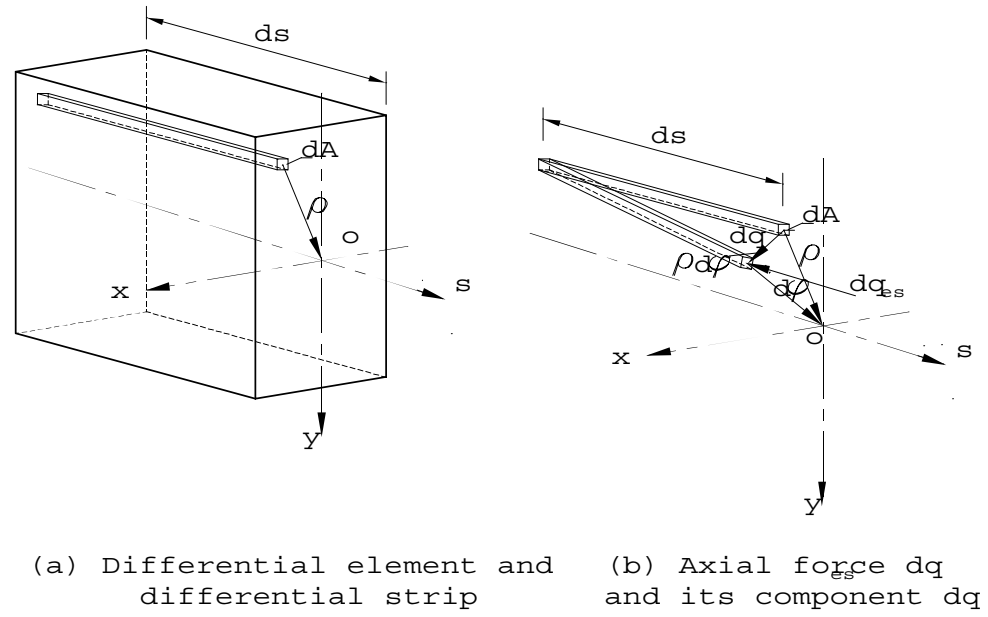

Figure 4. Torsional buckling of a column under uniform compression

\subsection{Flexural-Torsional Buckling of Arches}

When a pin-ended arch with a in-plane radial load $q$ acting at the centroid of the cross-section, uniformly distributed around the arch axis, the arch is subjected primarily to a uniform compressive force $Q=q R$ as shown in Figure 1(a). In this study, it is assumed that the directions of the radial loads do not change and remain parallel to their initial directions during deformation. Under this action, the arch may buckle out of the plane of loading and fail in a flexural-torsional mode. Similarly to the case of a column, in the buckled configuration of an arch, the axial compressive force $Q$ in the direction of the axis os has an axial compressive component $Q_{e s}=Q$ in the direction of the axis $o_{1} s_{1}$, and a lateral component $Q_{e x}=-Q u^{\prime}$ in the direction of the axis $o_{1} x_{1}$. The force $Q_{e s}$ 
also produces an external torsional moment action $M_{e s}$ about the axis $o_{1} s_{1}$ of the arch and is given by $[7,9]$

$$
M_{e s}=r_{0}^{2} Q_{e s} \kappa_{s}=r_{0}^{2} Q\left(\phi^{\prime}-\frac{u^{\prime}}{R}\right)
$$

while the total internal torsional resistance $M_{i s}$ can be obtained by combining the uniform and warping torsional resistances as $[9,11]$

$$
M_{i s}=-G J\left(\phi^{\prime}-\frac{u^{\prime}}{R}\right)+E I_{w}\left(\phi^{\prime \prime \prime}-\frac{u^{\prime \prime \prime}}{R}\right)
$$

In a column, the torsional action is in equilibrium with the torsional resistance as shown in the previous section. However, in an arch, the torsional action couples with the lateral bending action while the torsional resistance also couples with the lateral bending resistance. The resultant torsional moment at the cross-section is then given by

$$
M_{s}=M_{e s}+M_{i s}=r_{0}^{2} Q\left(\phi^{\prime}-\frac{u^{\prime}}{R}\right)-G J\left(\phi^{\prime}-\frac{u^{\prime}}{R}\right)+E I_{w}\left(\phi^{\prime \prime \prime}-\frac{u^{\prime \prime \prime}}{R}\right)
$$

which does not vanish.

The resultant lateral bending moment at the cross-section is equal to the lateral bending resistance $M_{i y}$ of the cross-section about the axis $o_{1} y_{1}$ and given by

$$
M_{y}=M_{i y}=-E I_{y} \kappa_{y}=-E I_{y}\left(u^{\prime \prime}+\frac{\phi}{R}\right) .
$$

For lateral moment equilibrium, it can be deduced from Figure 5 that

$$
\left(M_{y}+\mathrm{d} M_{y}\right)-M_{y}+2 M_{s} \sin \frac{\mathrm{d} \theta}{2}+Q_{e x} \mathrm{~d} s=\mathrm{d} M_{y}+\frac{M_{s}}{R} \mathrm{~d} s+Q_{e x} \mathrm{~d} s=0
$$

which leads to the differential equation of equilibrium for the lateral deformations as

$$
\frac{\mathrm{d} M_{y}}{\mathrm{~d} s}+\frac{M_{s}}{s} \frac{1}{R}+Q_{e x}=0
$$

For the torsional moment equilibrium, it can be shown from Figure 5 that

$$
\left(M_{s}+\mathrm{d} M_{s}\right)-M_{s}-2 M_{y} \sin \frac{\mathrm{d} \theta}{2}=\mathrm{d} M_{s}-M_{y} \frac{\mathrm{ds}}{R}=0
$$

which leads to the differential equation of equilibrium for the torsional deformations

$$
\frac{\mathrm{d} M_{s}}{\mathrm{~d} s}-\frac{M_{y}}{R}=0
$$




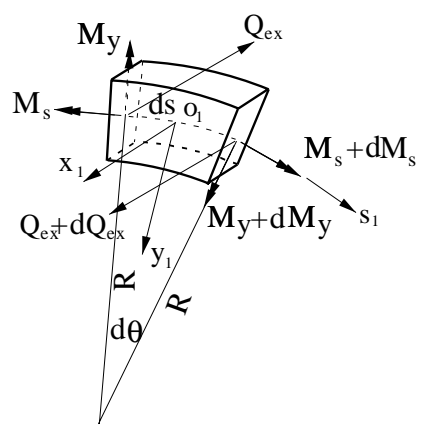

Figure 5. Buckling equilibrium of an arch element under uniform compression

It can be seen that the alternative method for the lateral buckling analysis for columns discussed in the previous section can be used for the flexural-torsional buckling analysis for arches. However, if the first method for the lateral buckling analysis of columns is employed for the flexural-torsional buckling of arches, the lateral bending action $M_{e y}=-Q u$ at the cross-section that is produced by the axial compressive force $Q$ will directly be used for the lateral bending equilibrium. Because of the coupling between the lateral bending and torsional actions, the lateral bending action $M_{e y}$ has a spurious component that will falsely contribute to the torsional action. Hence, to avoid the spurious torsional action, the alternative method is preferred and the lateral component $Q_{e x}$ of the axial compressive force $Q$ is used directly for the lateral bending equilibrium analysis of arches under uniform compression.

\subsection{Solutions for Flexural-Torsional Buckling}

Substituting $Q_{e x}=-Q u^{\prime}$ and the expressions for $M_{s}$ and $M_{y}$ given by Eqs (27)-(28) into Eqs (30) and (32) leads to the differential equations of equilibrium given by

$$
\left[E I_{y}\left(u^{\prime \prime}+\frac{\phi}{R}\right)\right]^{\prime}+G J\left(\phi^{\prime}-\frac{u^{\prime}}{R}\right) \frac{1}{R}-\left[E I_{w}\left(\phi^{\prime \prime}-\frac{u^{\prime \prime}}{R}\right) \frac{1}{R}\right]^{\prime}+Q\left[u^{\prime}-r_{0}^{2}\left(\phi^{\prime}-\frac{u^{\prime}}{R}\right) \frac{1}{R}\right]=0
$$

for the lateral deformations, and

$$
E I_{y}\left(u^{\prime \prime}+\frac{\phi}{R}\right) \frac{1}{R}-\left[G J\left(\phi^{\prime}-\frac{u^{\prime}}{R}\right)\right]^{\prime}+\left[E I_{w}\left(\phi^{\prime \prime}-\frac{u^{\prime \prime}}{R}\right)\right]^{\prime \prime}+\left[r_{0}^{2} Q\left(\phi^{\prime}-\frac{u^{\prime}}{R}\right)\right]^{\prime}=0
$$

for the torsional deformations.

The $n$-th mode buckled shapes of a pin-ended arch (Figure 1(c)) can be assumed to be given by

$$
\frac{u}{u_{m}}=\frac{\phi}{\phi_{m}}=\sin \frac{n \pi S}{S}
$$


which satisfy the boundary conditions at both ends of the arch $u_{0}=u_{S}=\phi_{0}=\phi_{S}=0$. Substituting Eq. (35) into Eqs (33) and (34) leads to the homogeneous equations

$$
\left[\begin{array}{ll}
k_{11} & k_{12} \\
k_{21} & k_{22}
\end{array}\right]\left\{\begin{array}{l}
u_{m} \\
\phi_{m}
\end{array}\right\}=\left\{\begin{array}{l}
0 \\
0
\end{array}\right\}
$$

where the coefficients $k_{11}, k_{12}, k_{21}$, and $k_{22}$ are given by

$$
\begin{aligned}
& k_{11}=\left[1+a_{n}^{2} b_{n}^{2}-\left(1+a_{n}^{2} b_{n}^{2} \frac{P_{y n}}{P_{s n}}\right) \frac{Q}{P_{y n}}\right] P_{y n} \\
& k_{12}=k_{21}=-\left(\frac{a_{n}}{b_{n}}+a_{n} b_{n}-a_{n} b_{n} \frac{P_{y n}}{P_{s n}} \frac{Q}{P_{y n}}\right) M_{y s n} \\
& k_{22}=\left(1+\frac{a_{n}^{2}}{b_{n}^{2}}-\frac{P_{y n}}{P_{s n}} \frac{Q}{P_{y n}}\right) r_{0}^{2} P_{s n}
\end{aligned}
$$

and in which the parameters $a_{n}$ and $b_{n}$ are defined by

$$
a_{n}=\frac{S}{n \pi R} \text { and } b_{n}=\frac{n \pi M_{y s n}}{P_{s n} S}
$$

and $M_{y s n}$ is the $n$-th mode elastic flexural-torsional buckling moment of the corresponding simply supported beam of length $S$ under uniform bending [2,15] given by

$$
M_{y s n}=\sqrt{r_{0}^{2} P_{y n} P_{s n}} .
$$

Eq. (36) has non-trivial solutions for $u_{m}$ and $\phi_{m}$ when the determinant of its coefficient matrix vanishes, i.e.

$$
k_{11} k_{22}-k_{12} k_{21}=0 \text {, }
$$

which leads to the equation for the elastic flexural-torsional buckling load of a pin-ended arch under uniform compression as

$$
\left(\frac{Q}{P_{y n}}\right)^{2}-\left[\left(1+\frac{a_{n}^{2}}{b_{n}^{2}}\right) \frac{P_{s n}}{P_{y n}}+\left(1-a_{n}^{2}\right)^{2} \frac{Q}{P_{y n}}\right]+\left(1-a_{n}^{2}\right)^{2} \frac{P_{s n}}{P_{y n}}=0
$$

which is the same as that of Pi and Bradford [9] using an energy method.

\subsection{Flexural Buckling or Torsional Buckling of Arches}

It can be seen from Eqs (33), (34) and (43) that if a pin-ended arch under uniform compression buckles out-of-plane, it will buckle in a flexural-torsional mode. This is different from a column under uniform compression which may buckle only in a flexural mode or in a torsional mode. 
However, if the torsional deformations of a pin-ended arch under uniform compression are fully prevented $\left(\phi=\phi^{\prime}=\phi^{\prime \prime}=\phi^{\prime \prime \prime}=0\right)$, the arch may buckle in a flexural mode and the differential equation of equilibrium for flexural buckling of the arch can be obtained from Eq. (33) by substituting $\phi=\phi^{\prime}=\phi^{\prime \prime}=\phi^{\prime \prime \prime}=0$ as

$$
\left(E I_{y} u^{\prime \prime}\right)^{\prime}-G J \frac{u^{\prime}}{R^{2}}+E I_{w} \frac{u^{\prime \prime \prime}}{R^{2}}+Q\left(u^{\prime}+r_{0}^{2} \frac{u^{\prime}}{R^{2}}\right)=0
$$

when $R \rightarrow \infty$, Eq. (44) becomes

$$
\left(E I_{y} u^{\prime \prime}\right)^{\prime}+Q u^{\prime}=0
$$

which is equivalent to Eq. (14) or (16) for the flexural buckling of a column under uniform compression.

By substituting the $n$-th mode buckling shapes given by Eq. (17) into Eqs (44), the flexural buckling load of an arch under uniform compression can be obtained as

$$
Q_{y n}=\frac{R^{2}}{R^{2}+r_{0}^{2}}\left(P_{y n}+\frac{r_{0}^{2}}{R^{2}} P_{s n}\right)
$$

which is higher than the $n$-th mode flexural buckling load $P_{y n}$ of a column under uniform compression [2,15] given by Eq. (18). When $R \rightarrow \infty$, Eq. (46) becomes the flexural buckling load of a column of length $S$

$$
Q_{y n}=P_{s n} .
$$

If the lateral deformations of a pin-ended arch under uniform compression are fully prevented ( $u=u^{\prime}=u^{\prime \prime}=u^{\prime \prime \prime}=0$ ), the arch may buckle in a torsional mode and the differential equation of equilibrium for torsional buckling of the arch can be obtained from Eq. (34) by substituting $u=u^{\prime}=u^{\prime \prime}=u^{\prime \prime \prime}=0$ as

$$
E I_{y} \frac{\phi}{R^{2}}-\left[G J \phi^{\prime}\right]^{\prime}+\left[E I_{w} \phi^{\prime \prime}\right]^{\prime \prime}+\left[r_{0}^{2} Q \phi^{\prime}\right]^{\prime}=0
$$

When $R \rightarrow \infty$, Eq. (48) becomes

$$
-\left[G J \phi^{\prime}\right]^{\prime}+\left[E I_{w} \phi^{\prime \prime}\right]^{\prime \prime}+\left[r_{0}^{2} Q \phi^{\prime}\right]^{\prime}=0
$$

which is equivalent to Eq. (22) for the torsional buckling of a column under uniform compression.

By substituting the $n$-th buckling shapes given by Eq. (23) into Eq. (48), the torsional buckling load of an arch under uniform compression can be obtained as

$$
Q_{s n}=\frac{E I_{y} a_{n}^{2}}{r_{0}^{2}}+P_{s n}
$$


which is higher than the $n$-th mode torsional buckling load of a column under uniform compression [2,15] given by Eq. (24). When $R \rightarrow \infty, a_{n} \rightarrow 0$ and Eq. (50) becomes the torsional buckling load of a column of length $S$

$$
Q_{s n}=P_{s n}
$$

\subsection{Comparisons}

When the radius of gyration of the cross-section $r_{0}$ is very small such as for a narrow rectangular section, the effects of the external torsional moment $M_{e s}$ given by Eq. (25) produced by the axial compressive force become very small compared with those of the external lateral force $Q_{e x}$ given by Eq. (13). In this case, the effects of the torsional moment $M_{e s}$, i.e. the terms in the differential equations of equilibrium (33) and (34) containing the factor $r_{0}^{2}$ can be ignored, and so the flexural-torsional buckling load reduces to

$$
\frac{Q}{P_{y n}}=\frac{\left(1-a_{n}^{2}\right)^{2}}{1+a_{n}^{2} / b_{n}^{2}}
$$

which is the same as that obtained by Timoshenko and Gere [2] for arches with a narrow rectangular section, and by Papangelis and Trahair [4], when energy methods were used.

A number of researchers [3-9] have used energy or virtual work methods to obtain closed form solutions for the flexural-torsional buckling load of arches under uniform compression. Solutions for the first mode flexural-torsional buckling load of arches under uniform compression given by Eq. (43) are compared with the solutions by other researchers [3-9,11] in Figure 6 for arches with an Australian steel cross-section 250UB37 [16] $\left(A=4750 \mathrm{~mm}^{2}, I_{x}=55.7 \times 10^{6} \mathrm{~mm}^{4}, I_{y}=5.66 \times\right.$ $\left.10^{6} \mathrm{~mm}^{4}, \quad J=158 \times 10^{3} \mathrm{~mm}^{4}, I_{w}=85.2 \times 10^{9} \mathrm{~mm}^{6}, E=200,000 \mathrm{MPa}, v=0.3\right)$ and with a length $S$ $=2000 \mathrm{~mm}$. It can be seen that there are some differences between the results. In particular, the result of Yoo [3] has a substantial discrepancy with the other results [4-9]. Although generally the energy or virtual work methods used by most researchers [4-9] were correct, the second order terms in the nonlinear strains differed from each other. In addition, the treatments for the external torsional moment were also somewhat different from each other. These may be the sources of small discrepancies among the solutions of [4-9]. Yoo [3] substituted curvature expressions for an arch directly into the strain energy and potential energy expressions that were derived for straight members, and this is probably the source of the large differences between Yoo's result and that of others. It is worth pointing out that Vlasov [11] in his study assumed that uniform compression in arches was produced by hydrostatic loads, although his result has been benchmarked by a number of researchers [3-9] as being the case for the radial loads discussed in this study. Vlasov [11] substituted the curvature $\kappa_{y}$ and twist $\kappa_{s}$ into the differential equations of equilibrium that were derived for straight members, and obtained the differential equations of equilibrium as

$$
E I_{y} \kappa_{y}^{\prime \prime}+G J \kappa_{s}^{\prime} \frac{1}{R}-E I_{w} \kappa_{s}^{\prime \prime \prime} \frac{1}{R}+Q \kappa_{y}=0
$$

for the lateral deformations, and

$$
E I_{y} \kappa_{y} \frac{1}{R}-G J \kappa_{s}^{\prime}+E I_{w} \kappa_{s}^{\prime \prime \prime}+r_{0}^{2} Q \kappa_{s}^{\prime}=0
$$


for the torsional deformations. It can be seen from Eqs. (53) and (54) that the coupling between the internallateral bending resistance and the internal torsional resistance was considered. However, the coupling term contributed by the external torsional moment $M_{e s}$ to the lateral bending deformations was missed in Eq. (53). In addition, direct substitution of curvature $\kappa_{y}$ leads to a lateral force term $Q \kappa_{y}$ in Eq. (53). By substituting $\kappa_{y}$ given by Eq. (11), $Q \kappa_{y}=Q\left(u^{\prime \prime}+\phi / R\right)$, which contains a lateral force $Q \phi / R$. In the present study and in most other studies [2-9], it is assumed that the directions of the radial uniformly distributed loads do not change but always remain parallel to the initial directions during buckling and so the lateral force $Q \phi / R$ does not exist for this load case. However, the directions of hydrostatic loads change during buckling. Hence, the lateral force $Q \phi / R$ does exist and may be understood as the contribution of the horizontal component of hydrostatic loads to the lateral resistance. It is therefore argued that the lateral force $Q \phi / R$ is the major source of the large differences of the result of Vlasov [11] from those of the other researchers [4-9].

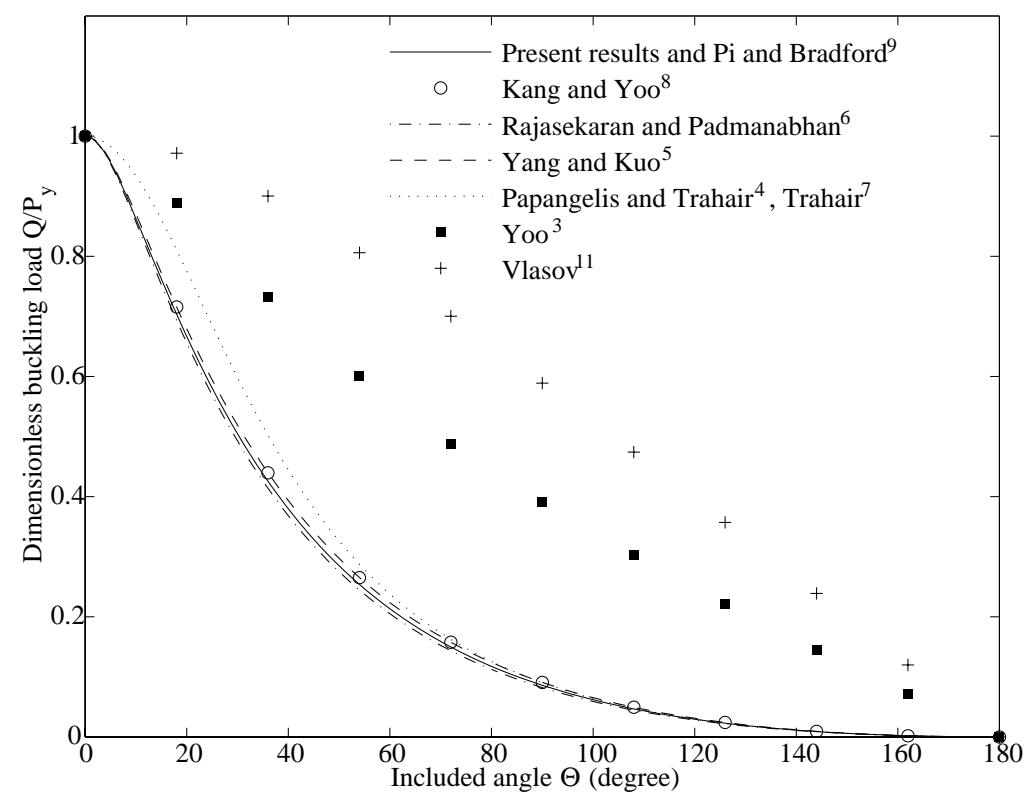

Figure 6. Comparisons of results for flexural-torsional buckling under uniform compression

It can also be seen from Figure 6 that the flexural-torsional buckling load of a pin-ended arch under uniform compression is lower than both the flexural buckling load and the torsional buckling load of a corresponding column.

\section{UNIFORM BENDING}

\subsection{Method of Timoshenko and Gere}

When an arch simply supported in-plane and pin-ended laterally is subjected to in-plane equal and opposite bending moments $M$ at both ends as shown in Figure 1(b), the arch is under uniform bending. Under this action, the arch may also buckle out of the plane of loading and fail in a flexural-torsional mode. Because the ends of the arch are free to move together and apart in the plane of loading for this special case, the arch can also be considered as a beam curved in elevation. In the buckled configuration, the in-plane uniform bending moment $M$ has the components: an in-plane bending moment $M_{e x}$ about the axis $o_{1} x_{1}$, a lateral bending moment $M_{e y}$ about the axis $o_{1} y_{1}$, and a torsional moment $M_{e s}$ about the axis $o_{1} s_{1}$ and given by 


$$
\left\{\begin{array}{l}
M_{e x} \\
M_{e y} \\
M_{e s}
\end{array}\right\}=\mathbf{R}^{T}\left\{\begin{array}{c}
M \\
0 \\
0
\end{array}\right\}=\left\{\begin{array}{c}
M \\
-M \phi \\
M u^{\prime}
\end{array}\right\}
$$

Timoshenko and Gere [2] studied the flexural-torsional buckling of a circular arch with a narrow rectangular cross-section under uniform bending. The warping moment resistance of a narrow rectangular cross-section is very small and so was ignored in their study. Timoshenko and Gere [2] considered equilibrium in the buckled configuration as shown in Figure 7. If the warping moment resistance is considered, the solution of Timoshenko and Gere [2] can be generalized in the following. For lateral equilibrium, the sum of the external bending action $M_{e y}$ and the internal bending resistance $M_{i y}$ vanishes, i.e.

$$
M_{e y}+M_{i y}=0
$$

Substituting $M_{e y}$ given by Eq. (55) and $M_{i y}$ given by Eq. (28) into Eq. (56) leads to the differential equation of equilibrium for lateral deformations given by

$$
M \phi+E I_{y}\left(u^{\prime \prime}+\frac{\phi}{R}\right)=0 .
$$

For torsional equilibrium, the sum of the external torsional action $M_{e s}$ and the internal torsional resistance $M_{i s}$ as vanishes, i.e.

$$
M_{e s}+M_{i s}=0 .
$$

Substituting $M_{e s}$ given by Eq. (55) and $M_{i s}$ given by Eq. (26) into Eq. (58) leads to the differential equation of equilibrium for torsional deformations as

$$
M u^{\prime}-G J\left(\phi^{\prime}-\frac{u^{\prime}}{R}\right)+\left[E I_{w}\left(\phi^{\prime \prime}-\frac{u^{\prime \prime}}{R}\right)\right]^{\prime}=0
$$

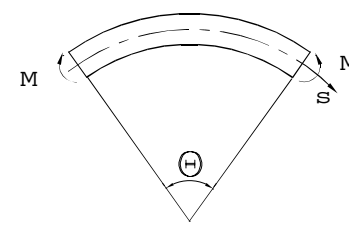

(a) Arch

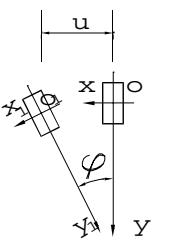

(b) Flexural-torsional buckling

Figure 7. Timoshenko's equilibrium method 


\subsection{Flexural-Torsional Buckling of Arches}

It can be seen that Timoshenko and Gere [2] treated the flexural-torsional buckling of an arch under uniform bending in the same way as for the flexural-torsional buckling of a beam under uniform bending. However, because of the curved shape, the lateral bending action necessarily couples with the torsion action and the lateral bending resistance necessarily couples with the torsion resistance, and the coupling terms were missed in the differential equations of equilibrium (57) and (59).

In the present study, the lateral and torsional equilibrium of a differential element of length $\mathrm{d} s$ in the buckled configuration is considered as shown in Figure 8. The resultant lateral bending moment $M_{y}$ at the cross-section is equal to the sum of the lateral bending action $M_{e y}$ given by Eq. (55) and internal bending resistance $M_{i y}$ given by Eq. (28), and given by

$$
M_{y}=M_{e y}+M_{i y}=-M \phi-E I_{y}\left(u^{\prime \prime}+\frac{\phi}{R}\right)
$$

which does not vanish.

The resultant torsional moment $M_{s}$ at the cross-section is given by combining the external torsional moment $M_{e s}$ given by Eq. (55) and internal torsional resistance $M_{i s}$ given by Eq. (26) as

$$
M_{s}=M_{e s}+M_{i s}=M u^{\prime}-G J\left(\phi^{\prime}-\frac{u^{\prime}}{R}\right)+E I_{w}\left(\phi^{\prime \prime \prime}-\frac{u^{\prime \prime \prime}}{R}\right)
$$

which also does not vanish.

For the lateral moment equilibrium, it can be shown from Figure 8 that

$$
\left(M_{y}+\mathrm{d} M_{y}\right)-M_{y}+2 M_{s} \sin \frac{\mathrm{d} \theta}{2}=\mathrm{d} M_{y}+M_{s} \frac{\mathrm{ds}}{R}=0
$$

from which the differential equation of equilibrium for lateral deformations becomes

$$
\frac{\mathrm{d} M_{y}}{\mathrm{~d} s}+\frac{M_{s}}{R}=0
$$

For the torsional moment equilibrium, it can be shown from Figure 8 that

$$
\left(M_{s}+\mathrm{d} M_{s}\right)-M_{s}-2 M_{y} \sin \frac{\mathrm{d} \theta}{2}=\mathrm{d} M_{s}-M_{y} \frac{\mathrm{ds}}{R}=0
$$

from which the differential equation of equilibrium for torsional deformations becomes

$$
\frac{\mathrm{d} M_{s}}{\mathrm{~d} s}-\frac{M_{y}}{R}=0
$$




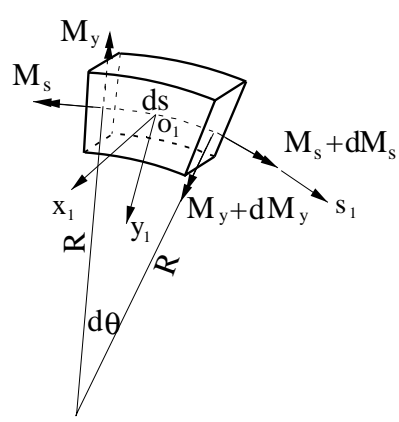

Figure 8. Buckling equilibrium of an arch element under uniform bending

\subsection{Solution for Flexural-Torsional Buckling Moment}

By substituting Eqs (60) and (61) into Eqs (63) and (65), the differential equations of equilibrium (63) and (65) become

$$
\left[E I_{y}\left(u^{\prime \prime}+\frac{\phi}{R}\right)\right]^{\prime}+G J\left(\phi^{\prime}-\frac{u^{\prime}}{R}\right) \frac{1}{R}-\left[E I_{w}\left(\phi^{\prime \prime}-\frac{u^{\prime \prime}}{R}\right)\right]^{\prime} \frac{1}{R}+(M \phi)^{\prime}-\frac{M u^{\prime}}{R}=0
$$

for the lateral deformations, and

$$
E I_{y}\left(u^{\prime \prime}+\frac{\phi}{R}\right) \frac{1}{R}-\left[G J\left(\phi^{\prime}-\frac{u^{\prime}}{R}\right)\right]^{\prime}+\left[E I_{w}\left(\phi^{\prime \prime}-\frac{u^{\prime \prime}}{R}\right)\right]^{\prime \prime}+M u^{\prime \prime}+\frac{M \phi}{R}=0
$$

for the torsional deformations. The $n$-th buckled shapes of the arch are also assumed to be given by Eq. (35). Substituting Eq. (35) into Eqs (66) and (67) yields the homogeneous equations (36). In this case, the coefficients $k_{11}, k_{12}, k_{21}$, and $k_{22}$ are given by

$$
\begin{aligned}
k_{11} & =\left(1+a_{n}^{2} b_{n}^{2}+a_{n} b_{n} \frac{M}{M_{y s n}}\right) P_{y n}, \\
k_{12}=k_{21} & =-\left(\frac{a_{n}}{b_{n}}+a_{n} b_{n}+\frac{M}{M_{y s n}}\right) M_{y s n}, \\
k_{22} & =\left(1+\frac{a_{n}^{2}}{b_{n}^{2}}+\frac{a_{n}}{b_{n}} \frac{M}{M_{y s n}}\right) r_{0}^{2} P_{s n} .
\end{aligned}
$$

In the same way as for arches under uniform compression, Eq. (36) has non-trivial solutions for $u_{m}$ and $\phi_{m}$ when the determinant of its coefficient matrix vanishes, i.e.

$$
k_{11} k_{22}-k_{12} k_{21}=0 \text {, }
$$


which leads to the equation for the elastic flexural-torsional buckling moment of an arch under uniform bending as

$$
\left(\frac{M}{M_{y s n}}\right)^{2}+\left(a_{n} b_{n}+\frac{a_{n}}{b_{n}}\right) \frac{M}{M_{y s n}}-\left(1-a_{n}^{2}\right)=0
$$

which is consistent with those of Vlasov [1] and of Rajasekaran and Padmanabhan [6].

\subsection{Comparisons}

The solution for the first mode elastic flexural-torsional buckling moment of in-plane simply supported and laterally pin-ended circular arches under uniform bending given by Eq. (71) is compared with the solutions obtained by other researchers [3-9,11] in Figure 9 for arches with an Australian steel cross-section 250UB37 [16] and a length $S=2000 \mathrm{~mm}$. The solutions of Papangelis and Trahair [4], Yang and Kuo [5], Trahair [7], Kang and Yoo [8] and Pi and Bradford [9] have small discrepancies from each other. The solution of Yoo [3] is significantly different from the other solutions[4-9]. Again, most of solutions [4-9] were correctly based on energy or virtual work methods, but different second order nonlinear strains lead to small discrepancies amongst them. Yoo [3] again used an analogy of arches with straight members, and so his solution resulted had large discrepancies with other solutions 4-9].

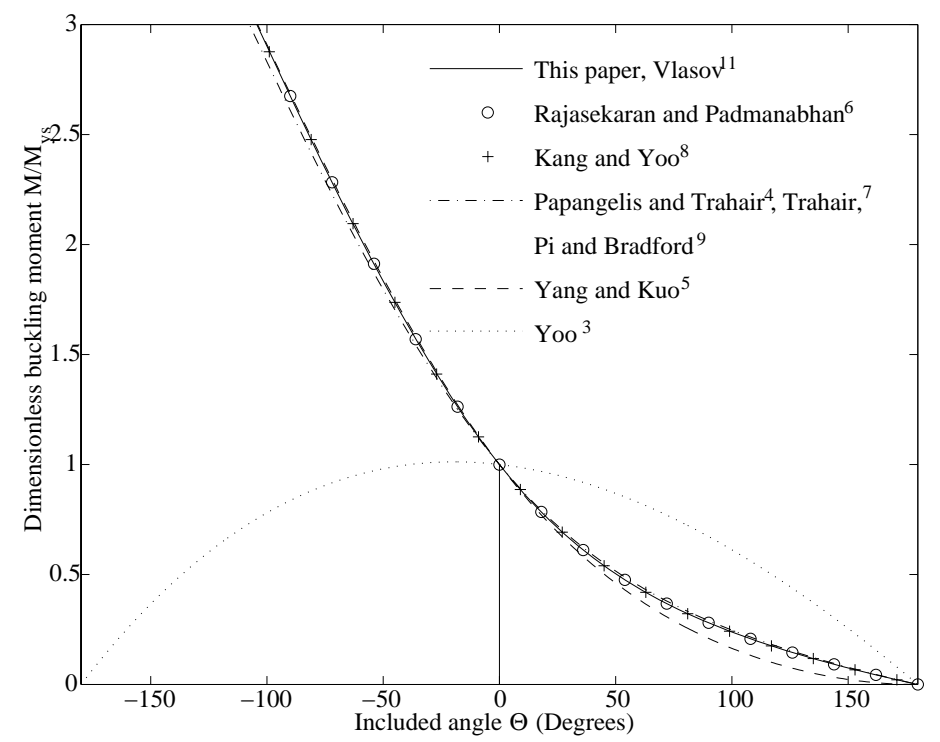

Figure 9. Comparisons of results for flexural-torsional buckling under uniform bending

Vlasov [11] substituted the curvature $\kappa_{y}$ and twist $\kappa_{s}$ into differential equations of equilibrium that were derived for the flexural-torsional buckling analysis of straight members, and considered the coupling between the internal lateral bending resistance and the internal torsional resistance. Because of the symmetric nature of the external lateral bending moment and resistance, and the torsional moment and resistance under uniform bending, Vlasov's method for the flexural-torsional buckling of arches under uniform bending is analogous to the present analysis. When the warping resistance is ignored, the solution of Eq. (71) reduces to that of Timoshenko and Gere [2]. However, as noted, since Timoshenko and Gere [2] considered equilibrium in the same way as for straight beams only at the cross-section, some coupling terms were lost in the differential equations of equilibrium. 
It can also be seen from Figure 9 that the flexural-torsional buckling moment of an arch under positive uniform bending as shown in Figure 1(b) is lower than that of a corresponding beam, while the flexural-torsional buckling moment of an arch under negative uniform bending (the end moments are opposite to those of Figure 1(b)) is higher than that of a corresponding beam.

\section{CONCLUSIONS}

This paper has used a rational static equilibrium approach to investigate the elastic flexural-torsional buckling of circular arches under uniform compression or under uniform bending. Solutions for the buckling moment and the buckling load have been obtained in closed form, and discrepancies among the existing solutions have been clarified.

It has been found that it is reasonable to use material curvatures and twist, rather than spatial curvatures and twist, for the flexural-torsional buckling analysis of arches. First order buckling deformations are sufficient for static equilibrium methods in the flexural-torsional buckling analysis of arches. When a rational static equilibrium approach is used for the flexural-torsional buckling analysis, equilibrium of a differential element should be considered and so couplings between the lateral bending action and resistance and the torsional action and resistance can be included in the differential equations of equilibrium. If the equilibrium is considered only at the cross-section, the coupling terms in the differential equations of equilibrium will be lost. A static equilibrium approach of substituting the lateral curvature and twist into the differential equations of equilibrium for the flexural-torsional buckling analysis of straight members is an analogy of arches with straight members, which should not be used for the flexural-torsional buckling analysis for arches since it may lead to incorrect results.

The flexural-torsional buckling load of a pin-ended arch under uniform compression is lower than both the flexural buckling load and the torsional buckling load of a corresponding column. A pin-ended arch under uniform compression may buckle in a flexural mode if torsional deformations are fully prevented, or may buckle in a torsional mode if lateral deformations are fully prevented. They are higher than their counterparts of a corresponding column. The flexural-torsional buckling moment of an arch under positive uniform bending is lower than that of a corresponding beam, while the flexural-torsional buckling moment of an arch under negative uniform bending is higher than that of a corresponding beam.

\section{ACKNOWLEDGEMENT}

This work has been supported by a Federation Fellowship, and a Discovery Project awarded to the second author, and a Discovery Project awarded to the first author, by the Australian Research Council.

\section{REFERENCES}

[1] Pi YL, Trahair NS, Out-of-plane inelastic buckling and strength of steel arches. Journal of Structural Engineering, ASCE 1998;124(2):174-183. 
[2] Timoshenko SP, Gere JM. Theory of elastic stability, 2nd Edition New York, USA: McGraw-Hill, 1961.

[3] Yoo CH. Flexural-torsional stability of curved beams. Journal of the Engineering Mechanics Division, ASCE 1982;108(EM6):1351-1369.

[4] Papangelis JP, Trahair NS. Flexural-torsional buckling of arches. Journal of Structural Engineering, ASCE 1987;113(4):889-906.

[5] Yang YB, Kuo SR. Effects of curvature on stability of curved beams. Journal of Structural Engineering, ASCE 1987;113(6):821-841.

[6] Rajasekaran S, Padmanabhan S. Equations of curved beams. Journal of Engineering Mechanics, ASCE 1989;115(5):1094-1111.

[7] Trahair NS. Flexural-torsional buckling of structures, London, UK: E \& FN Spon, 1993.

[8] Kang YJ, Yoo CH. Thin-walled curved beams. II: Analytical solutions for buckling of arches. Journal of Engineering Mechanics, ASCE 1994;120(10):2102-2125.

[9] Pi YL, Bradford MA. Elastic flexural-torsional buckling of continuously restrained arches. International Journal of Solids and Structures 2002;128(6):719-727.

[10] Pi YL, Bradford MA. Effects of approximations in analysis of beams of open thin-walled cross-section: 1. Flexural-torsional stability. International Journal for Numerical Methods in Engineering 2001; 51(7):757-772.

[11] Vlasov VZ. Thin-walled elastic beams, 2nd Edition. Jerusalem, Israel: Israel Program for Scientific Translation, 1961.

[12] Pi YL, Trahair NS. Nonlinear inelastic analysis of steel beam-columns. I: Theory. Journal of Structural Engineering, ASCE 1994;120(7):2041-2061.

[13] Pi YL, Bradford MA, Uy B. Nonlinear analysis of members curved in space with warping and Wagner effects. International Journal of Solids and Structures 2005; 42(11-12): 3147-3169.

[14] Gibbs J. Vector analysis. New York, USA: Dover, 1961.

[15] Trahair NS, Bradford MA, Nethercot DA. The behaviour and design of steel structures to BS5950, Third Edition - British, London, UK: E \& FN Spon, 2001.

[16] BHP. Hot rolled and structural steel products, 2000 Edition, Melbourne, Australia: BHP Co. Pty Ltd, 2000.

[17] Pi YL, Papangelis JP, Trahair NS. Prebuckling deformations and flexural-torsional buckling of arches. Journal of Structural Engineering, ASCE 1995;121(9):1313-1322. 
[Blank Page] 